\title{
Papers
}

\section{Cohort study on effects of parathyroid surgery on multiple outcomes in primary hyperparathyroidism}

Peter Vestergaard, Leif Mosekilde

\author{
Abstract \\ Objectives To assess the effects of surgery compared \\ with conservative treatment (no surgery) for primary \\ hyperparathyroidism. \\ Design Cohort study. \\ Setting Nationwide Danish cohort. \\ Participants 3213 patients, mean age 61 (SD 16) \\ years, with a diagnosis of primary \\ hyperparathyroidism between 1980 and 1999. 1934 \\ $(60 \%)$ underwent surgery and $1279(40 \%)$ were \\ treated conservatively. \\ Main outcome measures Occurrence of fractures, \\ osteoporosis, kidney or urinary tract stones, acute \\ myocardial infarction, angina pectoris, cardiac \\ arrhythmias, arterial hypertension, heart failure, \\ stroke, acute pancreatitis, stomach or duodenal ulcers, \\ muscle pain, malignant diseases, psychiatric disorders, \\ and mortality.
}

Results At diagnosis of primary hyperparathyroidism, patients who subsequently underwent surgery had a lower prevalence of previous fracture (odds ratio 0.64, $95 \%$ confidence interval 0.51 to 0.80 ), acute myocardial infarction $(0.59,0.42$ to 0.83$)$, stroke $(0.57$, 0.37 to 0.88$)$, psychiatric disorders $(0.54,0.31$ to 0.94$)$, and painful muscle disorders $(0.44,0.26$ to 0.76$)$, whereas kidney stones $(2.49,1.93$ to 3.23$)$ and acute pancreatitis $(2.77,1.33$ to 5.76$)$ were more prevalent. After diagnosis, the risks of fractures (hazards ratio $0.69,0.56$ to 0.84$)$ and gastric ulcers $(0.59,0.41$ to 0.84 ) were lower in patients treated surgically than those treated conservatively. Events involving kidney or urinary tact stones were more prevalent in patients treated surgically than patients treated conservatively (1.87, 1.30 to 2.68$)$. Mortality was lower in patients treated surgically $(0.65,0.57$ to 0.73$)$.

Conclusions Patients treated surgically for primary hyperparathyroidism have a lower prevalence of fractures and gastric ulcers than patients treated conservatively. The type of treatment had no effect on the occurrence of cardiovascular events.

\section{Introduction}

The high levels of parathyroid hormone in primary hyperparathyroidism cause demineralisation of bone and increase the risk of fracture. ${ }^{1}$ Hypercalciuria increases the risk of kidney and urinary tract stones. ${ }^{2}$ The high calcium and parathyroid hormone levels have been associated with atherosclerosis and acute myocardial infarction. ${ }^{3-7}$ Arterial hypertension, which does not regress with surgery, has also been reported in primary hyperparathyroidism, as have stomach and duodenal ulcers and acute and chronic pancreatitis. ${ }^{8-11}$

Most intervention studies on the effect of surgery in primary hyperparathyroidism are uncontrolled cohort or before and after trials comparing changes in left ventricular size, fracture risk, bone mineral density, and incidence of kidney stones. ${ }^{12}{ }^{12}$ They therefore have not evaluated the difference between surgically and non-surgically (conservatively) treated patients. One small randomised study of 53 patients evaluated the effects of surgery and conservative treatment on bone mineral density and found an increase in bone mineral density and quality of life scores in surgically treated patients. ${ }^{14}{ }^{15}$ We collected data on a large cohort of patients with primary hyperparathyroidism and evaluated the long term outcome of hard end points in surgically treated patients compared with conservatively treated patients.

\section{Materials and methods}

Data were obtained from the Danish national hospital discharge database. This central database contains information on patients admitted to hospital according to their unique identification number and type of disease (international classification of diseases, eighth and 10th revisions; the ninth revision was never used in Denmark). ${ }^{16}$ Any surgical procedures are also entered in the database. We were therefore able to obtain data on both diagnosis and admissions for concurrent illnesses in patients with primary hyperparathyroidism.

We retrieved information on patients with a first time diagnosis of primary hyperparathyroidism (ICD-8 codes: 252.00, 252.01, 252.03-05, 252.08, 252.09, and ICD10 codes: E21.0, E21.2, E21.3) for the period 1 January 1980 to 31 December 1999, and identified 3225 patients. Twelve $(0.4 \%)$ were excluded: 10 were not Danish citizens, precluding follow up; the identification number was incorrect in one; and one had emigrated. This left 3213 for analysis. We identified 1934 patients $(60 \%)$ who had undergone surgery and $1279(40 \%)$ who had not. Information was obtained on all hospital contacts for these 3213 patients from 1 January 1977 to 31 December 1999. Our primary end points of interest were fractures, osteoporosis, kidney and urinary tract stone events, acute myocardial
Department of Endocrinology and Metabolism C, Aarhus Amtssygehus,

Aarhus University Hospital, Aarhus, Denmark

Peter Vestergaard senior registrar

Leif Mosekilde professor of internal medicine

Correspondence to: $P$ Vestergaard,

Osteoporosis Clinic Aarhus

Amtssygehus, Tage Hansens Gade 2, DK-8000 Aarhus C, Denmark

p-vest@post4.tele.dk

bmj.com 2003;327:530

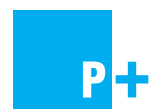

Additional tables appear on bmj.com 
infarction, angina pectoris, cardiac arrhythmias, arterial hypertension, cardiac insufficiency, stroke, stomach or duodenal ulcers, acute pancreatitis, poorly defined muscular pain, cancer, and psychiatric illness (psychoses and dementia) or neurotic disorders. We assessed whether any such contact had taken place before or after the date when primary hyperparathyroidism was diagnosed. We also obtained information on whether patients had died or emigrated during this period from the ministry of the interior. Total follow up time after diagnosis was 19565 (median 6.1) years. Before diagnosis the observation time was 47063 (median $15.9)$ years. The precision of the disease codes is generally considered to be high. ${ }^{16-18}$ Screening a random sample of 33 patients showed that the register based diagnosis of primary hyperparathyroidism was correct in $95 \%(\mathrm{n}=19)$ of surgically treated patients and $85 \%$ $(n=14)$ of conservatively treated patients. Screening a random sample of 35 patients with fracture diagnoses showed that $97 \%$ of register diagnoses agreed with hospital records. From a previous study the validity of a registered diagnosis of acute myocardial infarction was almost $90 \%{ }^{19}$

\section{Statistics}

Crude odds ratios were calculated for all outcomes. We used a Cox proportional hazard model to analyse differences between surgically and conservatively treated patients. We entered as potential confounders in the model, age at diagnosis of primary hyperparathyroidism for patients aged 50 or over compared with those under 50 , sex, and presence of the end point in question before the time of diagnosis. The presence of an end point before the time of diagnosis presents a special problem. In some cases, the patient may have more than one end point-for example, fractures occurring several times during follow up, and a fracture before diagnosis may be a risk factor for sustaining a new fracture after primary hyperparathyroidism was diagnosed. However, in other instances a prior end point may be a chronic disease for which the individual simply returns to receive medical attention (for example, cancer). We analysed the dataset both with the end points before the time of diagnosis and without. The results were similar for both approaches. We used logistic regression (likelihood ratio method) to evaluate difference in the prevalence of concurrent diseases at the time primary hyperparathyroidism was diagnosed, adjusted for age $(\geq 50 v<50$ years) and sex (female $v$ male). We computed the models with all vari-

Table 1 Characteristics of patients at time primary hyperparathyroidism was diagnosed stratified by surgery or conservative treatment (no surgery). Values are numbers (percentages) unless stated otherwise

\begin{tabular}{lccc} 
Variable & Surgery $(\mathbf{n}=\mathbf{1 9 3 4})$ & No surgery $(\mathbf{n}=\mathbf{1 2 7 9 )}$ & P value \\
\hline Mean (SD) age (years) & $58.3(15.2)$ & $64.2(17.4)$ & $<0.01^{*}$ \\
\hline Men & $500(26)$ & $293(23)$ & $0.06 \dagger$ \\
\hline Women & $1434(74)$ & $986(77)$ & \\
\hline Charlson indexł: & & $<0.01 \dagger$ \\
\hline 0 & $1385(72)$ & $852(67)$ & \\
\hline 1 & $259(13)$ & $199(16)$ & \\
\hline 2 & $204(11)$ & $142(11)$ & \\
\hline 3 & $59(3)$ & $53(4)$ & \\
\hline$\geq 4$ & $27(1)$ & $33(3)$ & \\
${ }^{*}$ Two sided $t$ test. & & \\
$\dagger \chi^{2}$ test. \\
$\neq 0=$ no comorbidity; $1-4=$ increasing comorbidity.
\end{tabular}

ables, entered using SPSS version 10.0. We compared the observed survival in the cohort of patients with that of the age matched and sex matched normal population using survival tables from the National Bureau of Statistics. ${ }^{20}$ The Charlson comorbidity index was used to assess survival in surgically treated compared with conservatively treated patients. ${ }^{21}$ This index comprises 19 comorbid conditions, including cardiovascular disease, cerebrovascular disease, peripheral artery disease, lung disease, liver disease, kidney diseases, dementia, cancer, and diabetes.

\section{Results}

Table 1 shows baseline characteristics at the time of diagnosis in all patients with primary hyperparathyroidism, stratified by treatment. The median time from diagnosis to surgery was 0.09 years (31 days), range $0-14$ years. Table 2 shows the presence of any end point before diagnosis and whether these risk factors were unevenly distributed among the groups. Table 3 shows the effect of surgery on the occurrence of a variety of end points after diagnosis. The risk of fractures was $31 \%$ (hazard ratio 0.69) lower in surgically treated patients than in conservatively treated patients whereas the risk of stomach and duodenal ulcers was $41 \%$ (0.59) lower. A history of any of the end points increased the risk of having a recurrent episode of that end point. More episodes of kidney stones occurred after diagnosis among those who had surgery than among those who did not. The results were not changed by including the Charlson comorbidity index as a confounder.

After adjustment for potential confounders, the risk of death was lower in surgically treated patients than in conservatively treated patients (hazards ratio $0.65,95 \%$ confidence interval 0.57 to 0.73 ; table 4 ). The risk of death was lower in patients with a history of kidney stones. Prior myocardial infarction, hypertension, cardiac failure, arrhythmia, cancer, fracture, and ulcer were associated with an increased risk of death (see table 4). The results were not changed by including the Charlson comorbidity index $(0.62,0.55$ to 0.70 for surgical compared with conservative treatment) instead of the confounders. Survival was higher in surgically treated patients than in conservatively treated patients (figure). In both groups the observed survival was significantly lower than in the general population. However, the relative loss of expected life time was higher among conservatively treated patients than among surgically treated patients (mean $20 \%$ or 2.7 years $v 14 \%$ or 2.1 years, $\mathrm{P}<0.01$ ) after adjustment for age and sex. The proportion undergoing surgery decreased with time: $66 \%$ of patients diagnosed from 1980 to 1989 underwent surgery compared with $56 \%$ of those diagnosed between 1990 and $1999(\mathrm{P}<0.01)$.

\section{Discussion}

Surgical treatment for primary hyperparathyroidism led to fewer fractures and episodes of gastroduodenal ulcers and lower mortality than conservative treatment. Surgery was not, however, associated with a change in risk of cardiovascular events. 
Table 2 Presence of end points before diagnosis of primary hyperparathyroidism stratified by surgery or conservative treatment (no surgery). Values are numbers (percentages) of patients unless stated otherwise

\begin{tabular}{|c|c|c|c|c|c|c|}
\hline \multirow[b]{2}{*}{ Variable } & \multirow{2}{*}{$\begin{array}{c}\text { Surgery } \\
(n=1934)\end{array}$} & \multirow{2}{*}{$\begin{array}{c}\text { No surgery } \\
(\mathrm{n}=1279)\end{array}$} & \multirow{2}{*}{$\begin{array}{l}\text { Crude odds ratio for } \\
\text { surgery v no surgery } \\
(95 \% \mathrm{Cl})^{\star}\end{array}$} & \multicolumn{3}{|c|}{ Odds ratio $(95 \% \mathrm{Cl})$} \\
\hline & & & & Adjusted for age† & Adjusted for sex & Adjusted for surgerył \\
\hline Fractures & $172(9)$ & $176(14)$ & $0.61(0.49$ to 0.76$)$ & 2.07 (1.49 to 2.87 ) & $1.14(0.86$ to 1.50$)$ & $0.64(0.51$ to 0.80$)$ \\
\hline Kidney stones & $297(15)$ & $83(7)$ & 2.61 (2.04 to 3.35$)$ & 0.59 (0.47 to 0.75$)$ & $0.49(0.39$ to 0.61$)$ & 2.49 (1.93 to 3.23$)$ \\
\hline Stroke & $38(2)$ & $46(4)$ & 0.54 (0.35 to 0.83$)$ & 6.37 (2.32 to 17.48$)$ & 0.64 (0.39 to 1.03$)$ & 0.57 (0.37 to 0.88$)$ \\
\hline Hypertension & $85(4)$ & $58(5)$ & $0.97(0.69$ to 1.36$)$ & 1.52 (0.98 to 2.38$)$ & $0.76(0.52$ to 1.11$)$ & 0.99 (0.70 to 1.39$)$ \\
\hline Acute myocardial infarction & $65(3)$ & $75(6)$ & 0.54 (0.39 to 0.76$)$ & 6.48 (3.00 to 13.97$)$ & $0.52(0.36$ to 0.75$)$ & 0.59 (0.42 to 0.83$)$ \\
\hline Angina & $59(3)$ & $50(4)$ & $0.77(0.53$ to 1.13$)$ & 4.98 (2.29 to 10.80$)$ & $0.56(0.37$ to 0.84$)$ & $0.81(0.55$ to 1.20$)$ \\
\hline Heart failure & $29(2)$ & $42(3)$ & 0.45 (0.28 to 0.72$)$ & $19.45(2.76$ to 137.17$)$ & $1.19(0.65$ to 2.19$)$ & $0.49(0.30$ to 0.79$)$ \\
\hline Arrhythmias & $55(3)$ & $50(4)$ & $0.72(0.49$ to 1.06$)$ & 6.05 (2.45 to 14.95$)$ & 1.12 (0.69 to 1.82$)$ & 0.78 (0.53 to 1.15$)$ \\
\hline Pancreatitis & $38(2)$ & $9(1)$ & 2.83 (1.41 to 5.69$)$ & 0.77 (0.41 to 1.47$)$ & $1.02(0.52$ to 2.00$)$ & 2.77 (1.33 to 5.76$)$ \\
\hline Ulcers & $99(5)$ & $56(4)$ & 1.18 (0.84 to 1.65$)$ & 2.68 (1.60 to 4.49$)$ & 0.85 (0.58 to 1.23$)$ & 1.24 (0.88 to 1.74$)$ \\
\hline Muscle pain & $23(1)$ & $34(3)$ & $0.44(0.26$ to 0.74$)$ & 1.25 (0.63 to 2.44$)$ & $0.72(0.40$ to 1.29$)$ & $0.44(0.26$ to 0.76$)$ \\
\hline Osteoporosis & $35(2)$ & $27(2)$ & 0.85 (0.51 to 1.42$)$ & 7.81 (1.90 to 32.06$)$ & 8.52 (2.08 to 34.91$)$ & 0.96 (0.57 to 1.59$)$ \\
\hline Cancer & $135(7)$ & $119(9)$ & 0.73 (0.57 to 0.95$)$ & 3.49 (2.21 to 5.51$)$ & 1.13 (0.82 to 1.55$)$ & 0.78 (0.60 to 1.01$)$ \\
\hline Psychiatric disorder & $23(1)$ & $29(2)$ & 0.52 (0.30 to 0.89$)$ & 2.35 (0.99 to 5.57$)$ & $0.71(0.39$ to 1.31$)$ & 0.54 (0.31 to 0.94$)$ \\
\hline Neuroses & $33(2)$ & $36(3)$ & $0.60(0.37$ to 0.96$)$ & 3.01 (1.53 to 5.94$)$ & 7.63 (3.30 to 17.65$)$ & $0.70(0.42$ to 1.15$)$ \\
\hline
\end{tabular}

Adjustment performed by logistic regression.

*Unadjusted for age and sex.

†Aged $\geq 50 v<50$ years at diagnosis.

‡Surgery $v$ no surgery.

\section{Advantages and disadvantages of study}

The major advantage of our study is that we looked at a population based representative sample, allowing assessment of multiple outcomes over an extended period. Our study was not randomised, but relied on observational data from an administrative system collected for other purposes. Another major limitation was the lack of information on important confounders such as smoking. Thus bias may have affected the outcomes significantly. Selection bias could have affected the results in several ways. It is unlikely that the population was selected, as all cases of primary hyperparathyroidism in Denmark are managed in hospital and thus are included in the register. Selection bias is, however, a significant factor in deciding who should have surgery and who should be managed conservatively.
Surgery is the treatment of choice in patients with symptoms of primary hyperparathyroidism. Guidelines recommend that patients with asymptomatic primary hyperparathyroidism undergo surgery when there is a high concentration of serum calcium $(>1$ $\mathrm{mg} / \mathrm{dl}$ above normal range), high urine calcium output, and organ involvement (reduced kidney function or reduced bone mineral density). ${ }^{22}$ Thus those selected for surgery are more likely to have advanced disease with symptoms in contrast with those selected for conservative management, who will often have asymptomatic disease with a low risk of any end point. This would underestimate any difference between surgical and conservative treatment. On the other hand, some patients may simply be too unwell to tolerate surgery, and this would mean that some of

Table 3 Presence of end points after diagnosis stratified by surgery or conservative treatment (no surgery) for primary hyperparathyroidism and test of effects of surgery versus no surgery. Values are numbers (percentages) unless stated otherwise

\begin{tabular}{|c|c|c|c|c|c|c|c|}
\hline \multirow[b]{2}{*}{ Variable } & \multirow[b]{2}{*}{ Surgery } & \multirow[b]{2}{*}{ No surgery } & \multirow{2}{*}{$\begin{array}{l}\text { Crude odds ratio* } \\
\qquad(95 \% \mathrm{Cl})\end{array}$} & \multicolumn{4}{|c|}{ Hazard ratio $(95 \% \mathrm{Cl}) \dagger$} \\
\hline & & & & Ageł & Sex & Prior event§ & Surgeryा \\
\hline Fractures & $235(12)$ & $170(13)$ & 0.90 (0.73 to 1.11$)$ & 1.90 (1.46 to 2.48 ) & 1.66 (1.26 to 2.17$)$ & 2.76 (2.14 to 3.55$)$ & 0.69 (0.56 to 0.84$)$ \\
\hline Kidney stones & $174(9)$ & $37(3)$ & 3.32 (2.35 to 4.68$)$ & 0.69 (0.52 to 0.91$)$ & 0.82 (0.61 to 1.09$)$ & 8.62 (6.51 to 11.43$)$ & 1.87 (1.30 to 2.68$)$ \\
\hline Stroke & $128(7)$ & $74(6)$ & 1.15 (0.86 to 1.55$)$ & 5.95 (3.49 to 10.15$)$ & 0.87 (0.63 to 1.21$)$ & 3.61 (2.03 to 6.41$)$ & 0.90 (0.68 to 1.21$)$ \\
\hline Hypertension & $96(5)$ & $35(3)$ & 1.86 (1.26 to 2.74$)$ & 0.98 (0.67 to 1.44$)$ & 0.81 (0.56 to 1.19$)$ & 7.41 (4.73 to 11.61$)$ & 1.30 (0.88 to 1.91$)$ \\
\hline $\begin{array}{l}\text { Acute myocardial } \\
\text { infarction }\end{array}$ & $98(5)$ & $53(4)$ & 1.23 (0.88 to 1.74$)$ & 3.83 (2.26 to 6.52$)$ & $0.57(0.40$ to 0.80$)$ & 5.11 (3.29 to 7.95$)$ & 0.96 (0.69 to 1.35$)$ \\
\hline Angina & $94(5)$ & $56(4)$ & $1.12(0.80$ to 1.57$)$ & 2.48 (1.59 to 3.88 ) & 0.45 (0.32 to 0.62$)$ & 12.80 (8.45 to 19.40$)$ & 0.84 (0.60 to 1.18$)$ \\
\hline Heart failure & $87(5)$ & $57(5)$ & 1.01 (0.72 to 1.42 ) & 6.19 (3.12 to 12.27$)$ & 1.01 (0.68 to 1.52$)$ & 10.25 (5.98 to 17.55$)$ & 0.81 (0.58 to 1.14) \\
\hline Arrhythmias & $99(5)$ & $44(3)$ & 1.51 (1.06 to 2.17 ) & 2.24 (1.40 to 3.60$)$ & 0.63 (0.44 to 0.91$)$ & 13.78 (8.97 to 21.15 ) & 1.16 (0.81 to 1.66$)$ \\
\hline Pancreatitis & $18(1)$ & $8(1)$ & 1.49 (0.65 to 3.42$)$ & 0.75 (0.33 to 1.71$)$ & 0.60 (0.26 to 1.36$)$ & 45.22 (19.94 to 102.53) & 0.82 (0.35 to 1.95$)$ \\
\hline Ulcers & $69(4)$ & $55(4)$ & 0.82 (0.57 to 1.18 ) & 2.31 (1.41 to 3.80 ) & 0.73 (0.49 to 1.08$)$ & 3.78 (2.28 to 6.25$)$ & 0.59 (0.41 to 0.84$)$ \\
\hline Muscle pain & $34(2)$ & $25(2)$ & 0.90 (0.53 to 1.51$)$ & 1.32 (0.71 to 2.45 ) & 1.41 (0.73 to 2.75$)$ & 3.51 (0.84 to 14.58$)$ & 0.68 (0.40 to 1.15$)$ \\
\hline Osteoporosis & $36(2)$ & $29(2)$ & 0.82 (0.50 to 1.34$)$ & 4.45 (1.59 to 12.44$)$ & 3.39 (1.22 to 9.42$)$ & 14.88 (8.13 to 27.24$)$ & 0.63 (0.38 to 1.03$)$ \\
\hline Cancer & $270(14)$ & $127(10)$ & 1.47 (1.18 to 1.84$)$ & 2.45 (1.84 to 3.28 ) & 0.69 (0.55 to 0.86$)$ & 5.17 (4.06 to 6.59$)$ & $1.11(0.90$ to 1.37$)$ \\
\hline Psychiatric dis. & $44(2)$ & $33(3)$ & 0.88 (0.56 to 1.39$)$ & 3.01 (1.53 to 5.94$)$ & 0.70 (0.42 to 1.15$)$ & 7.63 (3.30 to 17.65$)$ & 0.66 (0.42 to 1.04$)$ \\
\hline Neuroses & $20(1)$ & $18(1)$ & 0.73 (0.39 to 1.39$)$ & 0.46 (0.24 to 0.90$)$ & 1.75 (0.76 to 4.04$)$ & 12.06 (5.49 to 26.46 ) & 0.61 (0.32 to 1.16$)$ \\
\hline
\end{tabular}

Hazard ratios are mutually adjusted.

*Unadjusted for age and sex.

†Cox regression model.

$\ddagger$ Aged $\geq 50 v<50$ years at diagnosis.

§Sustaining new end point after diagnosis if event had already occurred before diagnosis.

१Surgery $v$ no surgery. 
Table 4 Variables of significance to risk of death during follow up after surgery or conservative treatment (no surgery) for primary parahyperthyroidism

\begin{tabular}{ll} 
Variable & Hazard ratio $(95 \%$ Cl) \\
\hline Surgery $v$ no surgery & $0.65(0.57$ to 0.73$)$ \\
\hline Age $(\geq 50 v<50$ years $)$ & $3.85(3.14$ to 4.72$)$ \\
\hline Sex (women $v$ men) & $0.70(0.61$ to 0.80$)$ \\
\hline Medical history: & $0.57(0.46$ to 0.71$)$ \\
\hline Kidney stones & $1.57(1.27$ to 1.93$)$ \\
\hline Cancer & $1.34(1.02$ to 1.74$)$ \\
\hline Myocardial infarction & $1.06(0.75$ to 1.49$)$ \\
\hline Angina pectoris & $2.21(1.56$ to 3.14$)$ \\
\hline Cardiac failure & $1.48(1.07$ to 2.03$)$ \\
\hline Arrhythmia & $1.82(1.40$ to 2.36$)$ \\
\hline Hypertension & $1.34(0.95$ to 1.88$)$ \\
\hline Stroke & $1.40(1.09$ to 1.79$)$ \\
\hline Stomach or duodenal ulcer & $0.97(0.57$ to 1.64$)$ \\
\hline Acute pancreatitis & $1.29(1.06$ to 1.57$)$ \\
\hline Fracture &
\end{tabular}

${ }^{*}$ Cox proportional hazard analysis. Hazard ratios are mutually adjusted. Results not changed by replacing preoperative conditions with Charlson index.

those managed conservatively actually have a higher risk of death than those undergoing surgery. It is also possible that some patients did not want to undergo surgery, particularly those with psychiatric disorders. A trend was, however, shown towards a decrease in occurrence of psychiatric disorders in surgically treated patients.

Information bias seems to pose a smaller problem for fractures and acute myocardial infarction, as the precision of diagnoses was high. Precise data for the validity of certain end points, such as stomach ulcers, were not available. The lower precision of the diagnoses in those not undergoing surgery would tend to underestimate any effect of surgery because some of the patients did not have primary hyperparathyroidism. Although many of the conditions studied are managed in hospital (for example, acute myocardial infarction, cardiac arrhythmias, fractures), hypertension, muscle pain, neuroses, and gastric ulcers are often managed by general practitioners and are therefore not reflected in the data presented here. This may lead to a significant underestimation of these types of comorbidity. A special feature of bias is confounding by indication, when patients with urinary tract stones were more likely to undergo surgery than those without

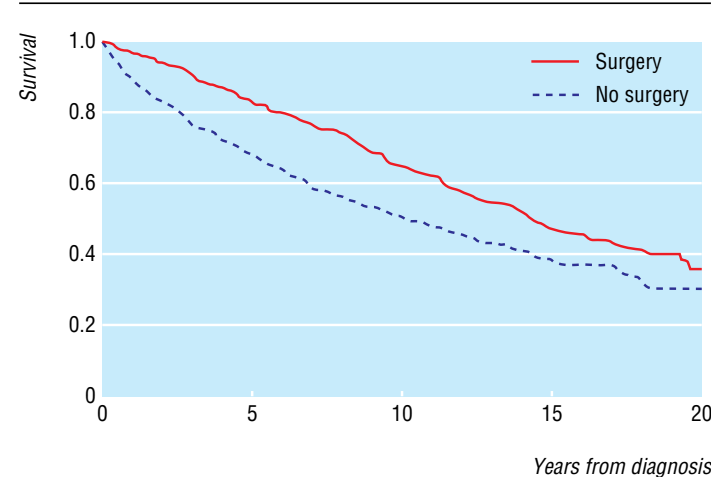

Kaplan-Meier plot of survival in surgically and conservatively treated patients with primary hyperparathyroidism. Death during follow up occurred in $591(31 \%)$ surgically treated patients and $474(37 \%)$ conservatively treated patients

\section{What is already known on this topic}

The risk of death is increased in primary hyperparathyroidism mainly due to an excess of deaths from cardiovascular causes

Bone mineral density increases after parathyroid surgery

Little is known on outcomes from cardiovascular events after surgery

\section{What this study adds}

Patients treated surgically for primary hyperparathyroidism have a lower risk of fractures and stomach and duodenal ulcers than those treated conservatively

Surgery was not associated with a change in the risk of cardiovascular events

The risk of kidney and urinary stones was higher both before and after surgery in those undergoing surgery

Mortality was lower in surgically treated patients

after the recommendations from international guidelines. Those who did not undergo surgery were older and had a higher mortality-that is, they may not have been offered surgery owing to their health and comorbidity.

\section{Effects of surgery on hard end points}

In contrast with the present study, we have presented the results of a study on patients only surgically treated for primary hyperparathyroidism. ${ }^{12}$ Although the risk of fracture was increased before surgery and returned to that of the general population within one year after surgery, it took 10 years or more for the risk of kidney stones to return to that of the general population. ${ }^{12}$ This may be caused by permanent damage to the urinary tract from strictures, persistent infections, or untreated stones. Although we tried to adjust for prior kidney stones, this may not have excluded patients with asymptomatic kidney stones, those whose pain was misdiagnosed as simple stomach pain, or those who did not at seek medical attention for the pain. Therefore, although some of the basis for recurrent stone formation (hypercalcaemia and hypercalciuria) is removed after parathyroidectomy, other factors may continue to induce stone formation. The risk of being admitted for gastric and duodenal ulcers decreased by $41 \%$ after surgery, although patients who underwent surgery tended to have more ulcers before diagnosis than those who did not have surgery. In accordance with our study, previous studies have shown that blood pressure is unchanged after surgery for primary hyperparathyroidism, probably indicating irreversible vascular or renal changes. ${ }^{9}$ The lack of effect on this important risk factor for other cardiovascular diseases may partly explain the absence of treatment effect on overall cardiovascular risk in our study.

The risk of death was smaller in surgically treated patients even after adjustment for several important confounders such as cardiovascular disease, cancer, 
and hypertension. Those not selected for surgery may have had a shorter expected lifespan than those selected for surgery. However, surgery did improve survival by decreasing serum calcium and serum parathyroid hormone levels. In our study those with prior kidney stones had a lower risk of death, a finding that agrees with another study. ${ }^{23}$ The reason for this remains unclear.

\section{Effect of time}

The incidence of primary hyperparathyroidism has changed significantly over time, because of increased access to measurements of serum calcium. ${ }^{24}$ The diagnostic criteria of increased serum calcium and serum parathyroid hormone levels have not changed over time, but milder cases are now diagnosed, and many of these do not require surgical management-reflected by the decrease in the proportion undergoing surgery. With the advent of milder cases the difference in the risk of end points between surgically and conservatively treated patients must be larger than if the cases were more balanced. However, the effects of surgery on these mild cases must be expected to be less than those on the more severe cases in our study.

Contributors: PV drafted the paper and analysed the data. PV and LM designed the study, interpreted the data, and prepared the final version of the paper. PV will act as guarantor for the paper.

Funding: None.

Competing interests: None declared.

1 Vestergaard P, Mollerup CL, Frøkjær VG, Christiansen P, Blichert-Toft M, Mosekilde L. Cohort study of risk of fracture before and after surgery for primary hyperparathyroidism. BMJ 2000;321:598-602.

2 Mollerup CL, Vestergaard P, Frøkjær VG, Mosekilde L, Christiansen P, Blichert-Toft M. Risk of renal stone events in primary hyperparathyroidism before and after parathyroid surgery: controlled retrospective follow up study. BMJ 2002;325:807-12.

3 Roberts WC, Waller BF. Effect of chronic hypercalcemia on the heart. An Roberts WC, Waller BF. Effect of chronic hypercalcemia on
analysis of 18 necropsy patients. Am J Med 1981;71:371-84.

4 Hedbäck G, Oden A, Tisell LE. The influence of surgery on the risk of death in patients with primary hyperparathyroidism. World J Surg 1991;15:399-405

5 Palmér M, Adami H-O, Bergstrøm R, Åkerström G, Ljunghall S. Mortality after surgery for primary hyperparathyroidism: a follow-up of 441 patients operated on from 1956-1979. Surgery 1987;102:1-7.
6 Hedback G, Oden A. Increased risk of death from primary hyperparathyroidism-an update [see comments]. Eur J Clin Invest

7 Leifsson BG, Ahren B. Serum calcium and survival in a large health screening program.J Clin Endocrinol Metab 1996;81:2149-53.

8 Daniels J, Goodman AD. Hypertension and hyperparathyroidism. Inverse relation of serum phosphate level and blood pressure. Am J Med 1983;75:17-23.

9 Sancho JJ, Roucho J, Riera-Vidal R, Sitges-Serra A. Long-term effects of parathyroidectomy for primary hyperparathyroidism on arterial hypertension. World J Surg 1992;16:732-6

10 Heath III H, Hodgson SF, Kennedy MA. Primary hyperparathyroidism: incidence, morbidity, and potential economic impact in a community. incidence, morbidity, and potent

11 Paloyan D, Simonowitz D, Paloyan E, Snyder TJ. Pancreatitis associated with primary hyperparathyroidism. Am Surg 1982;48:366-8.

12 Stefenelli T, Abela C, Frank H, Koller-Strametz J, Niederle B. Time course of regression of left ventricular hypertrophy after successful parathyroidectomy. Surgery 1997;121:157-61.

13 Christiansen P, Steiniche T, Brixen K, Hessov I, Melsen F, Heickendorff L, et al. Primary hyperparathyroidism: effect of parathyroidectomy on regional bone mineral density in Danish patients: a three year follow-up study. Bone 1999;25:589-95.

14 Rao D, Phillips ER, Divine GW, Talpos GB. Randomized controlled trial of surgery vs. no-surgery in patients with mild asymptomatic primary of surgery vs. no-surgery in patients with mild asymptomatic primary
hyperparathyroidism: final report. J Bone Miner Res 2000;15(suppl 1): hyperparathyroids. [Abstract.]

15 Talpos GB, Bone HG III, Kleerekoper M, Phillips ER, Alam M, Honasoge M, et al. Randomized trial of parathyroidectomy in mild asymptomatic primary hyperparathyroidism: patient description and effects on the SF-36 health survey. Surgery 2000;128:1013-20.

16 Andersen TF, Madsen M, Jørgensen J, Mellemkjær L, Olsen JH. The Danish National Hospital Register. Dan Med Bull 1999;46:263-8.

17 Jürgensen HJ, Frølund C, Gustafsen J, Mosbech H, Guldhammer B. [Registration of diagnoses in a national patient register. Preliminary assessment of the validity of the register] Ugeskr L e erer 1984;146.3303-8.

18 assoch J Jørensen J, Madsen M Rostaard K, Thornberg K, Poulsen TD. [The Danish National Patient Register: evaluation of data quality.] Ugeskr Lager 1995;157:3741-5.

19 Madsen M, Balling H, Eriksen LS. [The validity of the diagnosis of acute Madsen M, Balling H, Eriksen LS. [The validity of the diagnosis of acute
myocardial infarction in 2 registries: the Heart Registry compared to the National Patient Registry.] Ugeskr Laeger 1990;152:308-14.

20 Statistical yearbook 1998. Copenhagen: Danish Bureau of Statistics, 1998

21 Charlson ME, Pompei P, Ales KL, MacKenzie CR. A method of classifying prognostic comorbidity in longitudinal studies: development and validation. J Chron Dis 1987;40:373-83.

22 Bilezikian JP, Potts JT Jr, Fuleihan GE, Kleerekoper M, Neer R, Peacock M, et al. Summary statement from a workshop on asymptomatic primary hyperparathyroidism: a perspective for the 21st century.J Bone Miner Res 2002;17:N2-11.

23 Soreide JA, van Heerden JA, Grant CS, Yau Lo C, Schleck C, Ilstrup DM. Soreide JA, van Heerden JA, Grant CS, Yau Lo C, Schleck C, Ilstrup DM.
Survival after surgical treatment for primary hyperparathyroidism. Survival after surgical tre
Surgery 1997;122:1117-23.

24 Wermers RA, Khosla S, Atkinson EJ, Hodgson SF, O'Fallon WM, Melton LJ III. The rise and fall of primary hyperparathyroidism: a population-based study in Rochester, Minnesota, 1965-1992. Ann Intern Med 1997;126:433-40.

(Accepted 9 July 2003) 